\title{
Attenuation of Electromagnetic Wave Propagating Through Roofing Sheet: Aluminum, Zinc and Asbestos
}

\author{
Emmnauel I. Ugwu ${ }^{1,2,3}$, Stephen D. Songden ${ }^{2}$, Y. Y. Jabil ${ }^{2}$ \\ ${ }^{1}$ Department of Industrial Physics, Ebonyi State University, Abakaliki, Nigeria \\ ${ }^{2}$ Department of Physics, University of Jos, Jos, Nigeria \\ ${ }^{3}$ Department of Physical Science, Evangel University, Akaeze, Nigeria
}

Email address:

ugwuei@yahoo.com (E. I. Ugwu),ugwuei2@gmail.com (E. I. Ugwu)

\section{To cite this article:}

Emmnauel I. Ugwu, Stephen D Songden, Y. Y. Jabil. Attenuation of Electromagnetic Wave Propagating Through Roofing Sheet: Aluminum, Zinc and Asbestos. American Journal of Electromagnetics and Applications. Vol. 5, No. 1, 2017, pp. 7-13. doi: 10.11648/j.ajea.20170501.12

Received: March 15, 2017; Accepted: May 3, 2017; Published: July 13, 2017

\begin{abstract}
In this work, we study the attenuation characteristics of electromagnetic waves propagating through selected roofing sheets with lossy dielectric constant property. Wave equation relating the electromagnetic wave propagating through the materials was derived from Maxwell's equations considering all the parameters enshrined in the propagation constant such as the permeability, permittivity and dielectric constant of the Material. The wave equation was solved using method of separation of variable in 1-D and 2-D. An expression for Fresnel formula that was used in analysis of the relative amplitude for both reflection and transmission coefficients for parallel and perpendicular modes of polarization behaviour of the propagated waves was derive by considering small change in the refractive index of the materials. From the results of analysis, it was observed that the relative amplitudes which represent attenuation characteristics of the propagated wave in the materials for different incident and transmitted angles varied according to polarization modes and materials.
\end{abstract}

Keywords: Maxwell Equation, Electromagnetic Wave, Attenuation, Polarization, Propagation, Transmission, Dielectric Constant, Relative Amplitude, Analysis

\section{Introduction}

Various tools have been employed in studying and computing beam or field propagation in a medium with variation of small refractive index $[1,2]$ some researchers had employed beam propagation method based on diagonalization of the Hermetician operator that generates the solution of the Helmholtz equation in media with real refractive indices [3, 4], while some had used $2 \times 2$ propagation matrix formalism for finding the obliquely propagated electromagnetic fields in layered inhomogeneous un-axial structure [5]. Structure such as optical fibres, optical wave guides in the presence of electro-optical perturbation $[6,7,8]$. However, earlier before the, work had been going on veraciously on study of wave propagation in a stratified media, plasma and ionosphere [9, 10].

Van Roey in his work derived a general beam propagation relation in a number of specific cases along with the extensive simulation of wave propagation in variety materials while Matsunga et al had worked on wave propagation through tunnels with analysis of the effect of shape of wall on electromagnetic wave propagating around a building [11, $12]$.

Also, we have looked at the propagation of electromagnetic field through a conducting surface [2]. The behaviour of wave propagated through such material coupled with the influence of the dielectric function of the medium such on such material was analyzed. The effect of variation of refractive index of $\mathrm{F}_{\mathrm{e}} \mathrm{S}_{2}$ had also been carried out [13]. A close look on the concept made it clear to recognize the importance of the effect of the refractive index of the medium in the reality of the two velocity components that normally give rise to phase and group refractive indices as considered study of wave propagation $[14,15,16]$. Wave propagation in a metalized crystal, spatially inhomogeneous material have been carried in one and dimensions whereby different parameters regarding the material media of materials were considered. $[17,18]$

Study of wave propagation through a modeled thin with 
dielectric perturbation in which W.K.B approximation in conjunction with numerical approach were used has been carried out. [19, 20, 21]. Matsunga in their various works on propagation of waves in building expressed their experience on the wave propagating inside building and the source of it $[11$,$] and he had gone further to study electromagnetic wave$ propagating around building as well [12]

This work is based on a method that involves propagating an input field over a small distance through the roofing sheet medium and then by considering the small propagation distance as it relate to thickness of the material to analyze electromagnetic wave propagation through roofing sheets as it helps to understand the source of EM wave inside a building. In this case, we first derived Helmholtz Scalar wave equation from where the expression for the analysis of the profile of the field as it propagates in the medium is obtained. We also determine the transmission and reflection characteristics in terms of relative amplitude for various incidents and transmitted angles within the regions of electromagnetic wave spectrum with consideration given to the influence of the dielectric function on the propagated wave in the roofing sheets in conjunction with the polarization characteristic.

\section{Theoretical Procedure}

We consider Maxwell's equations for linear isotropic homogeneous dielectric medium of the roofing sheet with negligible conductivity in which the time factor $e^{\omega t}$ is suppressed. Under this consideration, the Maxwell's equations as regards the roofing sheet materials become

$$
\begin{gathered}
\nabla E_{s}=0 \\
\nabla . H_{s}=0 \\
\nabla X E_{s}=-\mu H_{s} \\
\nabla H_{s}=(\sigma+i \omega \varepsilon) E_{s}
\end{gathered}
$$

Where $\mu$ and $\varepsilon$ are permeability and permittivity of the material. Taking the curl of both sides of equation 3 with application of the vector identity and invocation of equation 1 and 4 we obtain

$$
\nabla E_{s}-\gamma^{2} E_{s}=0
$$

that is known as Helmholtz's equation in which $\gamma$ is the propagation constant given can be represented in the form

$$
\gamma^{2}=i \mu(\sigma+i \mu \varepsilon)
$$

Based on the complex nature of equation 6 , we write it as

$$
\gamma=\alpha+i \beta
$$

With which equation (7), we obtain what relate $\varepsilon, \beta$, with $\mu$ and $\sigma$.

As

$$
\sigma=\left\{\frac{\mu \varepsilon}{2} \sqrt{1+\left(\frac{\sigma}{\varepsilon}\right)^{2}-1}\right\}^{1 / 2}
$$

And

$$
\beta=\left\{\frac{\mu \varepsilon}{2}\left(\sqrt{1+\left[\frac{\sigma}{\varepsilon}+1\right]^{2}}\right)\right\}^{\frac{1}{2}}
$$

Since the assumed to be propagating along z-axis, we now equate 5 as

$$
\left[\nabla^{2}-\gamma^{2}\right] E_{x}^{(z)}=0
$$

With this, we now write thus

$$
\frac{\partial^{2} E_{z}}{\partial x^{2}}+\frac{\partial^{2} E_{z}}{\partial y^{2}}+\frac{\partial^{2} E_{z}}{\partial z^{2}}-\gamma^{2} E_{z}=0
$$

Being the 3-dimensional aspect of the equation

The solution of this equation using the method of separation of variable is of two forms

$$
E_{x}(z, t)=E_{o} e^{-\alpha z} \cos (\omega t-\beta z)
$$

and

$$
E(x, y, t)=\sum_{n=1}^{\infty}\left[2 \int_{0}^{l} \sin \frac{n \pi x}{l} f(x) d x+\sin \frac{n \pi y}{\omega} f(y) d y\right] e^{-\gamma^{2} t} \sin n \pi(x+y)
$$

Where $e^{-\sigma z}$ is the attenuation factor in Nepers per meter $(\mathrm{Np} / \mathrm{m})$ since $\sigma$ may not be completely ignored as it may be conductive in minute level.

Considering the refractive index of the material, the Helmholtz equation solution becomes

$$
E_{x}(z)=E_{o}(z) \exp -i \Delta n \beta_{o} z
$$

Where $\Delta n$ denotes change in refractive index of the material

Fourier transforming equation (13) gives

$$
\frac{\partial^{2} E(z)}{\partial z^{2}}=2 i n_{o} \frac{\partial E_{o}^{\prime}}{\partial z}-v E_{o}^{\prime}+\beta_{o}^{2} \partial \bar{n}^{2} E_{o}^{\prime}
$$

$E_{o}^{\prime}$ and $\partial \hat{n}$ denote the transform. $E_{o}, \partial \hat{n}^{2}$ denote convolution while $v$ is the spatial frequency.

The discretized counterpart of equation (14) is of the form 


$$
\left[\nabla^{2}-2 i n_{o} k_{o}\right] E_{o}=M E_{o}
$$

Where

$$
E_{o}^{\prime}=E_{o}^{\prime}(v, z)
$$

and

$M=\operatorname{Diag}\left(v_{1,}^{2} v_{2}^{2} \ldots . v_{N}^{2}\right)-\beta_{o} D$

$\mathrm{N}$ represents the number of discretized points. With this in mind, the formal solution to the differential matrix equation (15) for forward propagating wave becomes

$$
E^{\prime}(v, z)=\exp (-i 2 S) E^{\prime}(v, z)
$$

where $S=\left(n_{o}^{2} k_{o}^{2} I-M\right)^{1 / 2}$. I and $\mathrm{S}$ are the identity and Hermitian matrix respectively which can be diagonalized by using unitary matrix of its eigenvector as

$$
\hat{n}^{\dagger} S \hat{n}=S_{d}
$$

Finally, the solution of the Scalar Helmholtz equation becomes

$$
E^{\prime}(v, z)=\hat{n} \exp \left(-i 2 S_{d}\right) \hat{n}^{\dagger} E^{\prime}(v, z)
$$

$\exp \left(-i 2 S_{d}\right)$ can be used for the deduction of the expression of matrix relation in the case of normal and oblique incident for wave in the isotropic medium.

In order to analyze the behaviour of the wave as it propagates through the material's medium, we assume the medium to be uniaxial in which the optical axis is arbitrarily oriented in space of a cartesian co-ordinate system whose components depend on the thickness of the material which is assumed to be in line with the working co-ordinate. In principles it is considered that the structure of this nature is invariant in the direction of propagation and as such the field shape at the end of the propagation distance is visualized. Once the incident field is prescribed, in this case we neglect all the influence of reflection on the transmitted wave in order to enable us analyze the propagated wave profiles without the influence of reflected wave. (Ugwu et al 2012) For the analysis, of them propagating wave in the material, we assume the material to have a negligible conductive characteristic, with the a dielectric constants $\varepsilon$ independent of propagation distance within the layer of the medium which is visualize to be invariant in its optical parameters base on the solution of equation (11)., we can consider special case of Jones Matrix and isotropic medium with reference to normal incidence wave in an isotropic medium

$$
S(z)=\left(\begin{array}{cc}
\cos \theta_{i} & -\sin \theta_{i} \\
\sin \theta_{i} & \cos \theta_{i}
\end{array}\right)
$$

This represents Jones Matrix at normal incidence which can be extended to oblique incidence as well.

Base on the interfacial reflection which is unavoidable during propagation in the first medium, not withstanding its negligence in the onset of the derivation, matrix is introduced on the Fresnel Formulae to enable us consider the influence of the interfacial reflection.

$$
E_{z}(z, o)=\left(\begin{array}{ll}
E_{n_{1}} & x \\
E_{n_{1}} & y
\end{array}\right)=\left(\begin{array}{cc}
E_{n_{1}} & \cos \theta_{2} \sin \theta_{1} \\
E_{n_{1}} & \sin \theta_{2}
\end{array}\right)
$$

Also by considering the interfacial reflection into the second isotropic medium which is roofing sheet with refractive index, $n_{2}$ the transmission matrix for incident wave from the first medium with refractive $n_{1}$ can be written as $T\left(n_{1}, n_{2}\right)$

Invariably this is expressed as

$$
T\left(n_{1} n_{2}\right)=\left(\begin{array}{cc}
T_{11}\left(n_{1}, n_{2}\right. & 0 \\
0 & T_{22}\left(n_{1}, n_{2}\right)
\end{array}\right)
$$

Where $T_{11}$ and $T_{22}$ are the Fresnel formulae for the components that are parallel to and the components that are perpendicular to the plane of incidence (Ugwu et al 2012) respectively which are given as

$$
\begin{gathered}
T_{11}\left(n_{1}, n_{2}\right)=\frac{2 n_{1} \cos \theta_{2}}{n_{2} \cos \theta_{1} \cos \theta_{1} n_{2} \cos \theta_{2}} \\
T_{22}\left(n_{1}, n_{2}\right)=\frac{2 n_{1} \cos \theta_{1}}{n_{2} \cos \theta_{1} n_{2} \cos \theta_{2}}
\end{gathered}
$$

With this term we obtain the relative amplitude of the waves which is the wave amplitude in the medium of the material as a function of the initial amplitude of the propagating waves we consider two expected polarization modes that would generally be experienced as the wave propagates.

In 2-D the solution of the plane of propagating through the material can be spelt out as

$$
E_{z}(x, y, z)=X(x) Y(y) Z(z)
$$

$$
\begin{aligned}
& E(x, y, t)=\left(p \cos \frac{\lambda}{c} x+Q \sin \frac{\lambda}{c} x\right)\left[U \cos \frac{\lambda}{c} y+V \sin \frac{\lambda}{c} y\right] \varepsilon e^{-\lambda^{2} t} \\
& E(x, y, t)=\sum_{n=1}^{x}\left[2 \int_{0}^{l} \sin n \pi x / l f(x) d x+\sin n \pi y / \omega\right] f(y) d y
\end{aligned}
$$




$$
\lambda^{2}(x . y)=\frac{2 n^{2} \pi^{2} \mathcal{\varepsilon}}{\left(l^{2}+\omega^{2}\right) \sigma \rho}
$$

Where $\mathrm{n}=1,2,3$,

\section{Results / Discussion}

Fig. 1, 2 and 3 represent one dimensional wave profile for aluminum roofing sheet while fig. 4,5 and 6 are that of Zinc. Fig. 7, 8 and 9 represent that of asbestos. Two dimensional profiles are pictured in figures 10, to fig 16 . From the general observation, it is seen that that the attenuation factor as enshrined in the equation $12 \mathrm{a}$ and $12 \mathrm{~b}$ affected the behaviour of propagated wave profiles for the entire roofing sheets though there were no clear distinct characteristic as displayed by the profiles of different sheets for both 1-D and 2-D cases.

For Transmission and reflection characteristics which is the main part of the analysis that would showcase the attenuation characteristic nature these materials, change in the refractive index of the material was involved in the wave equation in order to enable the discretization representation of Fourier transformed Helmholtz equation for the purpose for the purpose of obtaining the Hermitician matrix which was used generate expression for the Transmission matrix for the incident wave from the first medium, $T\left(n_{1}, n_{2}\right)$ which invariably involves Fresnel formula that that is that main tool for the analysis of the relative amplitudes, transmission, reflection and the polarization characteristics of the propagated wave. From the tables; table 1, 2 and 3 which represent the data for all these parameters for Zinc, asbestos and aluminum where relative amplitude behaviour and polarization modes were displayed, it was seen that the relative amplitude for transmitted wave in parallel mode, $T_{I I}$ for zinc roofing sheet is highest when compared to aluminum and asbestos for the same incident and transmitted wave apart from $90^{\circ}$ incident angle where they all have the same value. Invariably, in the case of reflection, the relative amplitude for both parallel and perpendicular modes of polarization appeared to be highest for aluminum followed by zinc roofing sheet. However, it is noted that for the transmitted wave in case of perpendicular polarization modes all the relative amplitudes for all the incident and transmitted angles are negative

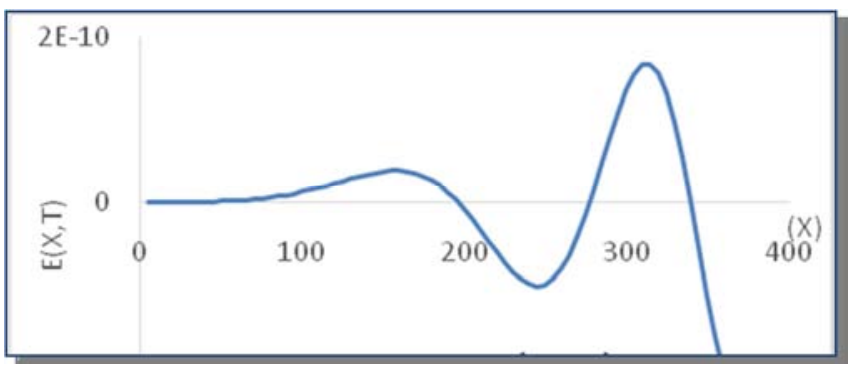

Figure 1. Graph of $E(x, t)$ as a function of position $(x)$ when the thickness is $0.9 \mathrm{~mm}$ for Aluminum.

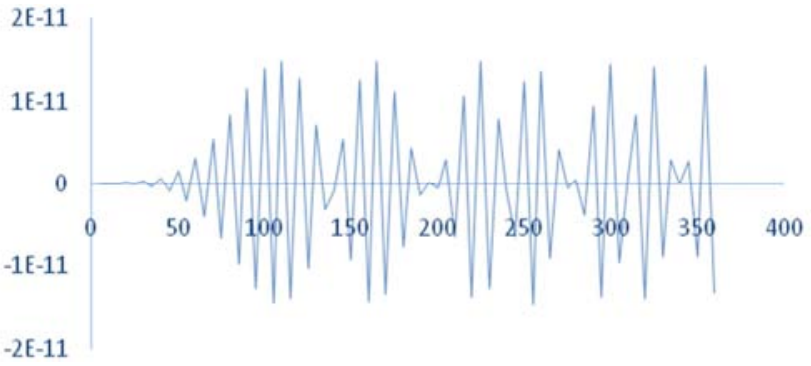

Figure 2. Graph of $E(x, t)$ as a function of position $(x)$ when the thickness is $0.5 \mathrm{~mm}$ for Aluminum.

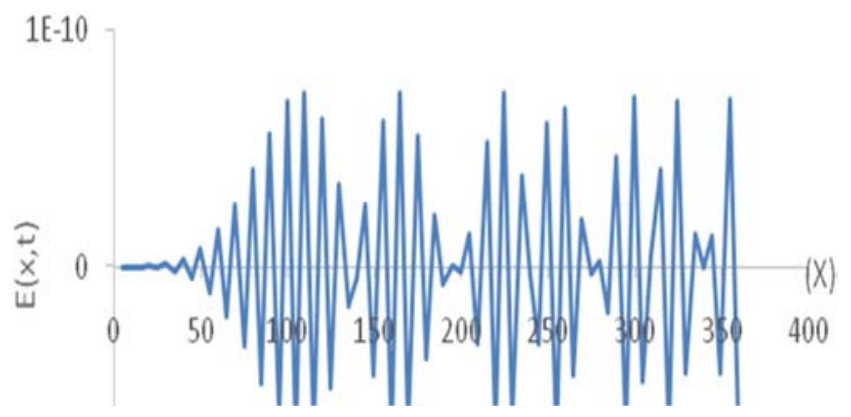

Figure 3. Graph of $E(x, t)$ as a function of position(x) when the thickness is $0.1 \mathrm{~mm}$ for Aluminum.

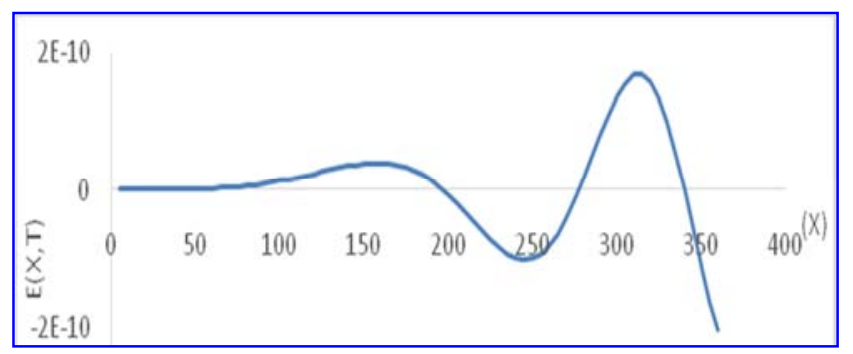

Figure 4. Graph of $E(x, t)$ as a function of position $(x)$ when the thickness is $0.9 m m$ for Zinc.

$(\mathrm{x})$

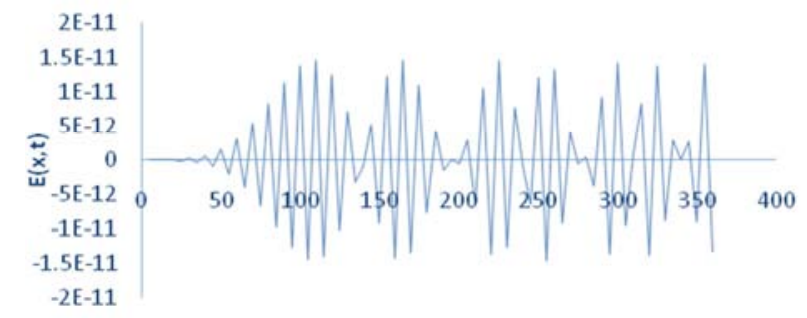

Figure 5. Graph of $E(x, t)$ as a function of position(x) when the thickness is $0.5 \mathrm{~mm}$ for Zinc.

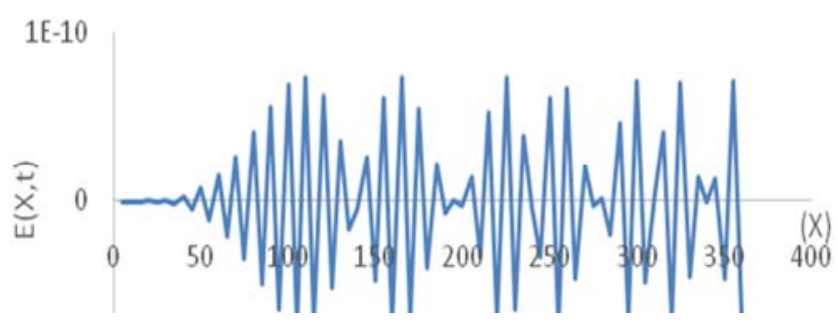

Figure 6. Graph of $E(x, t)$ as a function of position $(x)$ when the thickness is $0.9 \mathrm{~mm}$ for Zinc. 


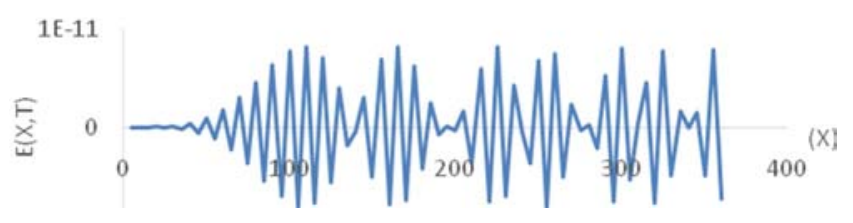

Figure 7. Graph of $E(x, t)$ as a function of position $(x)$ when the thickness is $0.9 \mathrm{~mm}$ for Asbestos.

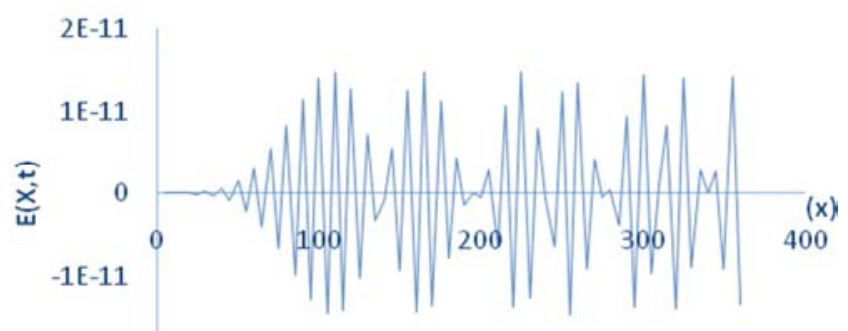

Figure 8. Graph of $E(x, t)$ as a function of position(x) when the thickness is $0.9 \mathrm{~mm}$ for Asbestos.

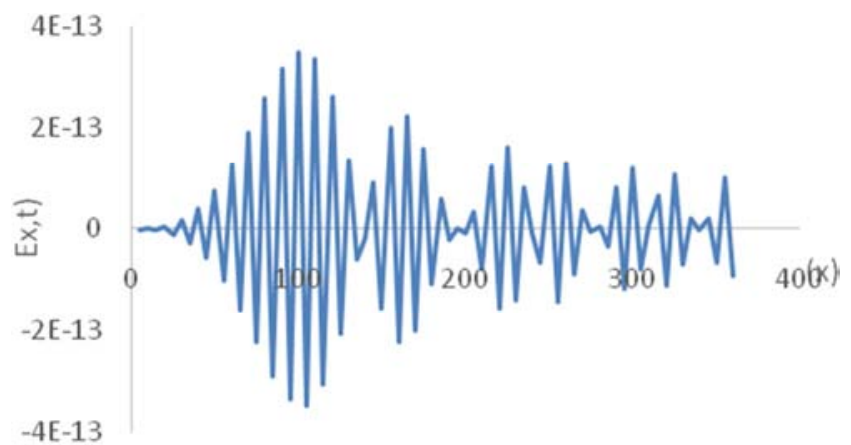

Figure 9. Graph of $E(x, t)$ as a function of position( $(x)$ when the thickness is $0.9 \mathrm{~mm}$ for Asbestos.

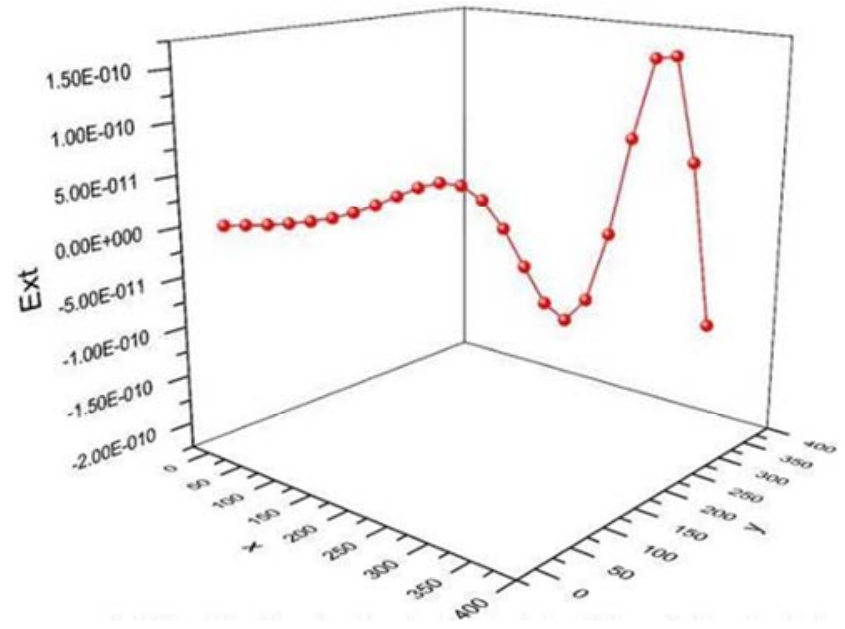

Figure 10. Graph $E(x, y, t)$ as a function of position $(x, y)$ when thickness is $0.9 \mathrm{~mm}$ for aluminum.

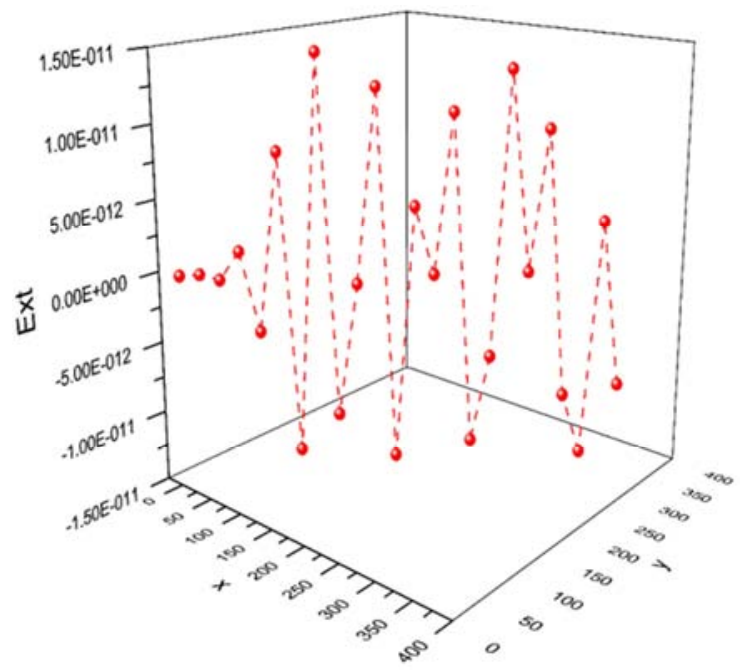

Figure 11. Graph $E(x, y, t)$ as a function of position $(x, y)$ when thickness is $0.5 \mathrm{~mm}$ for aluminum.

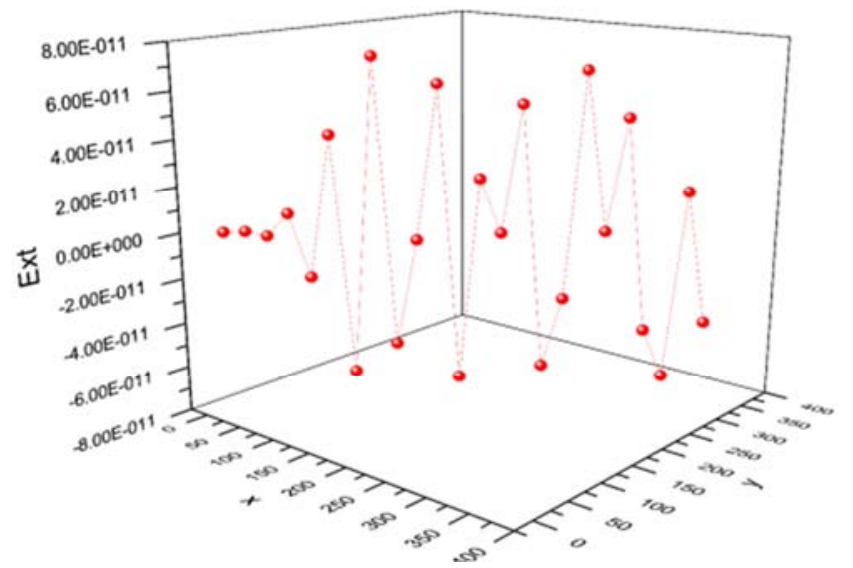

Figure 12. Graph E(x,y,t) as a function of position $(x, y)$ when thickness is $0.1 \mathrm{~mm}$ for aluminum.

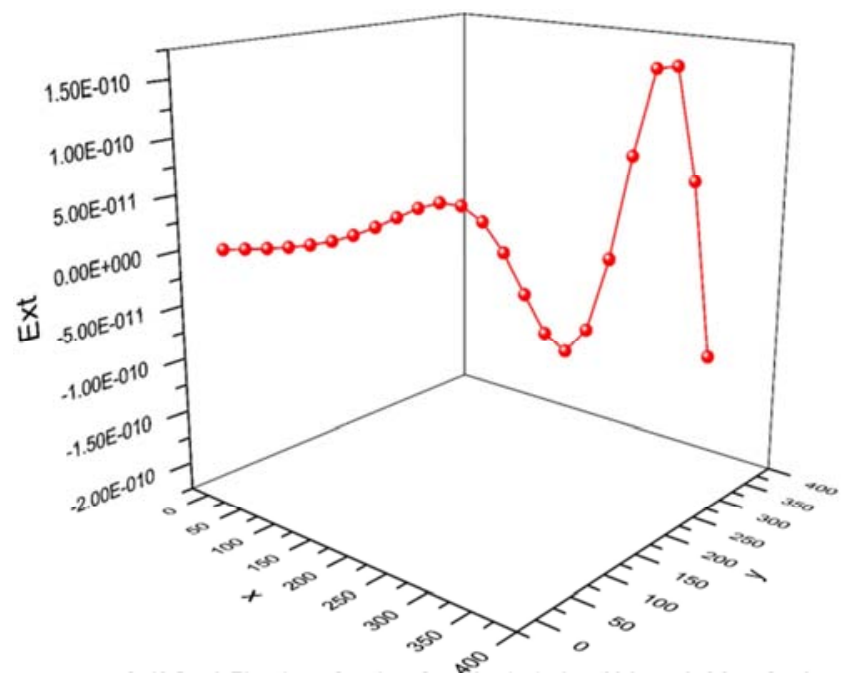

Figure 13. Graph $E(x, y, t)$ as a function of position $(x, y)$ when thickness is 0.9 mm for zinc. 


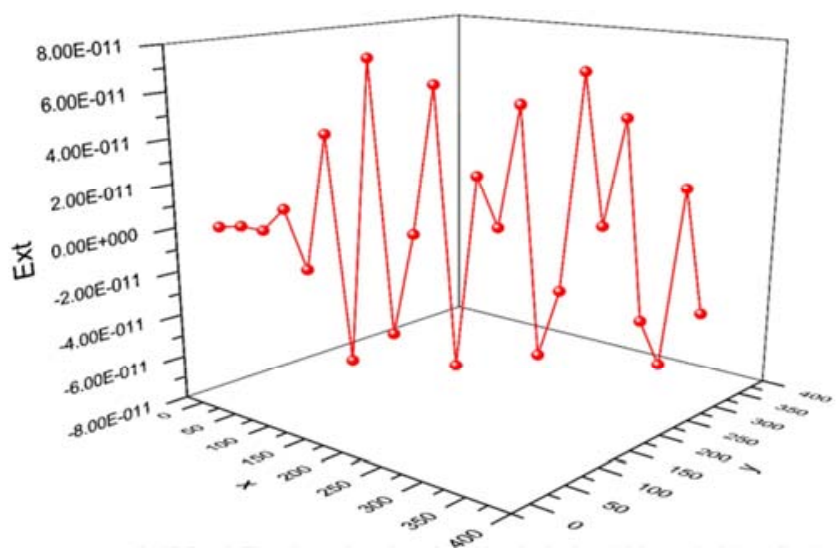

Figure 14. Graph $E(x, y, t)$ as a function of position $(x, y)$ when thickness is $0.1 \mathrm{~mm}$ for zinc.

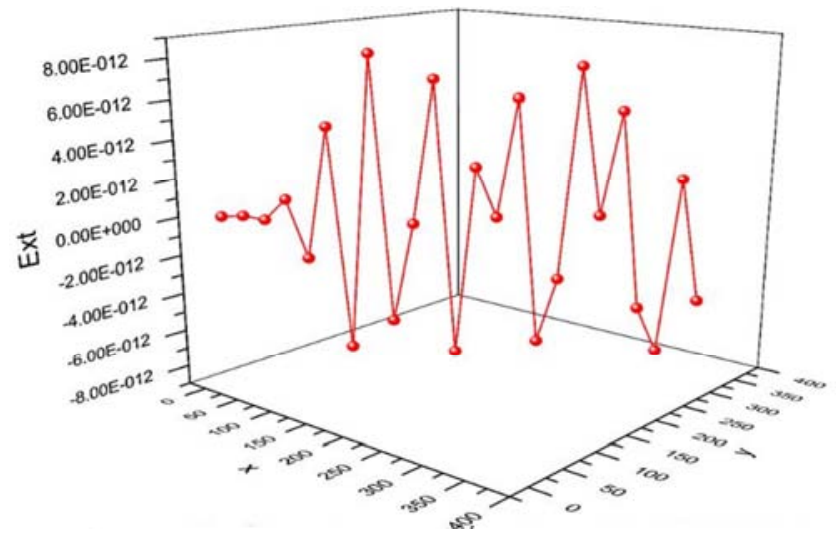

Figure 15. Graph $E(x, y, t)$ as a function of position $(x, y)$ when thickness is $0.9 \mathrm{~mm}$ for asbestos.

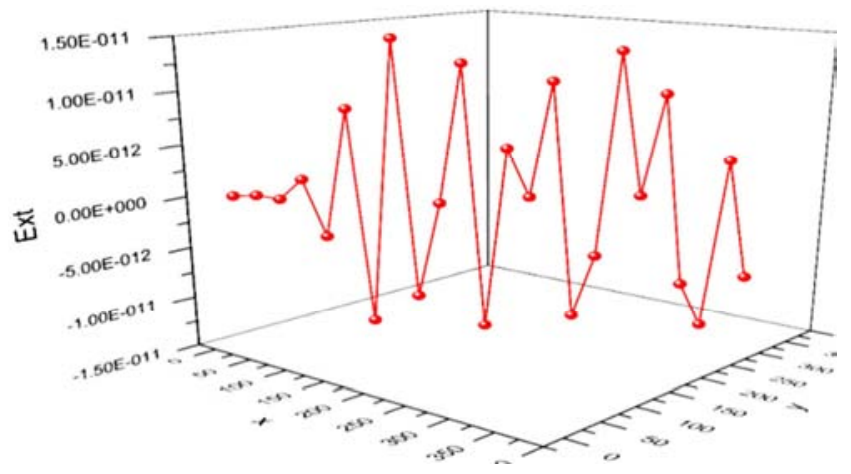

Figure 16. Graph $E(x, y, t)$ as a function of position $(x, y)$ when thickness is $0.5 \mathrm{~mm}$ for asbestos.

Table 1. The relative amplitude for transmission and reflection co-efficient for different incident and Transmitted angles for Zinc roofing sheet.

\begin{tabular}{clllll}
\hline $\boldsymbol{\theta}_{\boldsymbol{i}}$ & $\boldsymbol{\theta}_{\boldsymbol{t}}$ & $\boldsymbol{T}_{11}$ & $\boldsymbol{R}_{11}$ & $\boldsymbol{T}_{\perp}$ & $\boldsymbol{R}_{\perp}$ \\
\hline $0^{0}$ & $0^{0}$ & 0.489 & 1.489 & 0.000 & 1.489 \\
$15^{0}$ & $9.5^{0}$ & 0.497 & 1.466 & -0.032 & 1.481 \\
$30^{0}$ & $18.6^{0}$ & 0.523 & 1.391 & -0069 & 1.454 \\
$45^{0}$ & $27^{0}$ & 0.572 & 1.248 & -0.182 & 1.396 \\
$60^{0}$ & $33^{0}$ & 0.661 & 0.989 & -0.430 & 1.269 \\
$75^{0}$ & $36.9^{0}$ & 0.800 & 0.583 & -1.015 & 0.971 \\
$90^{0}$ & $38.1^{0}$ & 1.000 & 0.000 & -2.916 & 0.000 \\
\hline
\end{tabular}

Table 2. The relative amplitude for transmission and reflection co-efficient for different incident and transmitted angles for asbestos roofing sheet.

\begin{tabular}{clllll}
\hline $\boldsymbol{\theta}_{\boldsymbol{i}}$ & $\boldsymbol{\theta}_{\boldsymbol{t}}$ & $\boldsymbol{T}_{11}$ & $\boldsymbol{R}_{\boldsymbol{I I}}$ & $\boldsymbol{T}_{\perp}$ & $\boldsymbol{R}_{\perp}$ \\
\hline $0^{0}$ & $0^{0}$ & 0.373 & 0.687 & -2.191 & 0.687 \\
$15^{0}$ & $9.5^{0}$ & 0.382 & 1.354 & -0.014 & 1.364 \\
$30^{0}$ & $18.6^{0}$ & 0.411 & 0.645 & -0.063 & 1.338 \\
$45^{0}$ & $27^{0}$ & 0.468 & 0.583 & -0.165 & 1.269 \\
$60^{0}$ & $33^{0}$ & 0.572 & 0.468 & -0.384 & 0.937 \\
$75^{0}$ & $36.9^{0}$ & 0.743 & 0.282 & -0.867 & 0.829 \\
$90^{0}$ & $38.1^{0}$ & 1.000 & 0.000 & -2.191 & 0.000 \\
\hline
\end{tabular}

Table 3. The relative amplitude for transmission and reflection co-efficient for different incident and transmitted angles for Aluminum roofing sheet.

\begin{tabular}{llllll}
\hline $\boldsymbol{\theta}_{\boldsymbol{i}}$ & $\boldsymbol{\theta}_{\boldsymbol{t}}$ & $\boldsymbol{T}_{11}$ & $\boldsymbol{R}_{11}$ & $\boldsymbol{T}_{\perp}$ & $\boldsymbol{R}_{\perp}$ \\
\hline $0^{0}$ & $0^{0}$ & 0.544 & 2.000 & 0.000 & 1.544 \\
$15^{0}$ & $9.5^{0}$ & 0.552 & 1.520 & -0.016 & 1.537 \\
$30^{0}$ & $18.6^{0}$ & 0.575 & 1.439 & -0.071 & 1.512 \\
$45^{0}$ & $27^{0}$ & 0.620 & 1.286 & -0.189 & 1.458 \\
$60^{0}$ & $33^{0}$ & 0.701 & 1.014 & -0.453 & 1.338 \\
$75^{0}$ & $36.9^{0}$ & 0.825 & 0.591 & -1.093 & 1.047 \\
$90^{0}$ & $38.1^{0}$ & 1.000 & 0.000 & -2.978 & 0.000 \\
\hline
\end{tabular}

\section{Conclusion}

From this study, it is observed that electromagnetic wave propagating through the roofing sheets is found to have the same behaviours in their profile displayed in both 1-D and 2$\mathrm{D}$ characteristics. In the case of the relative amplitudes for both the transmission and reflection co-efficient, it observed that they depicted different characteristic which should be as a result of their different in their material properties such conductivity/ dielectric constants. Zinc and Aluminum displayed higher transmission co-efficient than the asbestos for parallel mode of polarization implying that higher quantity of the propagating electromagnetic wave is transmitted in the house. Though with higher reflection coefficient, the inner room of a house roofed with any of this roofing sheet is relatively hotter when compared with that of asbestos whose dielectric property acts as a polarizer/analyzer and as such will attenuated the polarized transmitted wave depending on the state of the polarized wave. Though now there may be still strayed wave propagating inside which invariably make the room still warm, which is due to the fact that some types of state of polarization is not completely exterminated which may be relatively smaller in the case of asbestos than in other two roofing sheets and this is why asbestos is till preferred in roofing of a house than zinc and aluminum.

\section{References}

[1] Feit, M. D \& Flecks, J. A. (1979), Light propagation in graded-index Optical fibres. Asppl. Optics, 17, 3990-3998.

[2] Ugwu, E. I., Uduh, P. C,. \& Agbo, G. A,. (2007) Wave Propagation in Non-homogeneous Thin Film of slowly varying Refractive index; W. K. B Solution Model. PJST, 8 (2) $246-251$. 
[3] Fleck, J. A., Morris, J. R., \& Feit M. D. (1976) TimeDependent Propagation of high energy laser beam through the Atmosphere, Appl. Phy., 10, 129- 160.

[4] Thylen, L. \& Lee, C. M. (1992) Beam Propagation Method based on matrix Diagonalization, J. Op. Sc A., 9 (1) 142- 146.

[5] Ong, H. L., (1993) $2 \times 2$ Propagation matrix for electromagnetic waves propagating obliquely in layered inhomogeneous uni- axial media J. Opt. Soc. America 10 (2) 203-393.

[6] Van-Roey, J., Donk, V., \& Lagasse J P. E. (1991) Beam Propagation; Analysis and Assessment, J. Opt. Soc Am., 71 803-810.

[7] Yevick, D., \& Glasner, M, (1990), Forward Wide Candle Light Propagation in Semiconductor Rib Wave guide, Opt Lett., 15, 174- 176.

[8] Kim, L. Gustafson, T. K G., \& Thylen, L. (1990), An Analysis of Quantum Confide Structures using Beam Propagation Method, Appl. Lett., 7. 285-287.

[9] Wait, J. R., (1970) Electromagnetic Wave in Stratified Media Second Edition Pergamon Press.

[10] Ginzbury, V. I., (1967), Propagation of Electromagnetic waves in Plasma Oxford Press.

[11] Matsunga M., Matsunga, T \& Sueyshi, T. (2009) An analysis of the effect of wall shape on electromagnetic waves propagating around buildings, Proceeding of the $39^{\text {th }}$ Microwaves Conference, pp 990-993.

[12] Matsunga, T., Uchida, K., and Kim, K. (1996) Electromagnetic Wave propagating in 2-D Tunnels with fundamental junction IEICE Transaction on Communication, Japanese Edition, 79 (7) 399-406.

[13] Ugwu, E. I., (2005) Effect of the Electric Conductivity of Thin film on Electromagnetic Wave Propagation, JICCOTECH Maiden Ed., 121-127.

[14] Valanju, P. M. R., Walser, M., and Valanju, P. A. (2002) Wave Reflection in Negative Index Media Always Positive and very Inhomogeneous Phys, Rev. Lett., 90.187401.

[15] Pentry, J. B., and Smith, D. R. (2003) Comment on Wave Refraction in Negative Index Media always positive and very inhomogeneous Phys. Lett., 229703.

[16] Cox, P. A (1978), Electronic Structure and Chemitry of Solid, Oxford Press.

[17] Ugwu, E. I., Eke, V. O. C., and Onyekachi, O. (2012) Electromagnetic Wave propagation in Spatially Inhomogeneous mThin film medium, Academy Publish, 197209).

[18] Alshits, V. I, Lyubimov, V. N., and Radowicz, A. (2011) Electromagnetic Waves in Crystals metallized Boundaries, Wave propagation. Intech Publisher, Chapter 3.

[19] Sankur., H. and Sothwell, W. H. (1984) Appl. Opt., 33. 2720.

[20] Menon, S., Sa, Q., and Grobe, I. (1981) Phy. Rev., E (67) 46619 (17) Ugwu, E. I., (2011) Wave Propagation in Dielectric Medium, Thin Film Medium, Wave Propagation, Intech. Publisher, Chapter 7.

[21] Matsunga, M. and Matsunga, T. (2011), Electromagnetic Waves Propagating Around Buildings, Wave Propagation, Intech Publisher, 553-570. 